\title{
Dor e funcionalidade na atenção básica à saúde
}

\author{
Pain and functionality in primary health care
}

$M$ atheus de Sousa $M$ ata ${ }^{1}$

Fabrícia Azevêdo da Costa ${ }^{1}$

Túlio Oliveira deSouza ${ }^{1}$

Ádala Nayana de Sousa M ata ${ }^{1}$

Jaqueline Fernandes Pontes ${ }^{1}$

${ }^{1}$ UniversidadeFederal do Rio Grande do Norte. Campus Universitário UFRN . Av. Senador Salgado Filho $s / n-$, Lagoa Nova. 59072-970 Natal RN mthmata@gmail.com
Abstract M usculoskeletal pain and functionality are linked to the individual health and, thus, influence the mode of interaction between individuals and between them and the environment that surrounds them. However, there are not many studies that question such health issues concerning the population in general, especially when we look at these issues from the basic care point of view. The aim of this article is to present initial data on the subject of pain and functionality in individuals with musculoskeletal complaints enrolled in a primary care unit and prompt a discussion on how complete the services provided by these units are. This study was carried out in a primary care unit and various households, among subjects aging 20 years or older. A functionality assessment tool and a Visual Analogue Scale were applied, and social and demographic issues were also considered. By using these tools, it was made an attempt to evaluate the profile of individuals in association with their degree of musculoskeletal pain and the degree of functionality in the activities of their daily lives, ages and occupations. We found a predominance of women in both groups: $84.37 \%$ and $81.25 \%$, respectively. Nearly half of all peoplewith musculoskeletal pain were housewives, and the average age between groups was over 55.

Key words Primary care, Pain, Functional ability, Musculoskeletal disorders
Resumo A dor osteoarticular ea funcionalidade são elementos ligados à saúde dos indivíduos e, portanto, influenciam o modo de interação entre si e entre eles e o meio ambiente que os cerca. Contudo, não são muitos os estudos que levantam essas questões de saúde na população em geral, sobretudo quando aproximamos o olhar da atenção básica sobre elas. Esteartigo tem o objetivo de fornecer dados iniciais sobre dor efuncionalidade nos indivíduos com queixa osteoarticular em uma população adscrita a uma unidade básica de saúde (UBS) e suscitar o debate sobre integralidade da atenção nesse serviço. 0 trabalho descriti vo foi realizado na UBS $(\mathrm{n} 1=64)$ e nos domicílios ( n2=48), entreindivíduos a partir dos 20 anos de idade. Foi aplicado um instrumento de avaliação de funcionalidadee a Escala Visual Analógica de dor, e foram exploradas questões sociodemográficas. Com esses instrumentos, buscou-se avaliar o perfil dos indivíduos em relação ao seu grau de dor musculoesquelética, o grau de funcionalidade nas atividades da vida diária, idade e ocupação. N os resultados, encontramos uma predominância do sexo feminino nos dois grupos, $84,37 \%$ e $81,25 \%$, respectivamente. Quase metade das pessoas com dor musculoesquelética eram donas de casa, e a média de idade entre os grupos estava acima dos 55 anos.

Palavras-chave Atenção primária. Dor, Capacidade funcional, Distúrbios musculoesqueléticos 


\section{Introdução}

A partir da Declaração de Alma-Ata, a atenção primária passa a ter um conjunto de conceitose fundamentos que a guiam até os dias atuais. Já neste documento, observam-se conceitos como universalização do acesso, baixo custo, participação social, primeiro contato dos indivíduos com o sistema de saúde, intersetorialidade, integração dos níveis de assistência, educação em saúdee problemas principais das comunidades ${ }^{1,2}$.

M esmo utilizando uma nomenclatura diferente, no Brasil a atenção primária, referida mais frequentemente como atenção básica, é baseada nos mesmos conceitos contidos na Declaração de Alma-Ata ${ }^{3}$, a despeito das particularidades da organização do Sistema Ú nico de Saúde (SUS). Acrescentam-se aos princípios e diretrizes da organização da saúde no país termos como integralidade, territorialização, humanização e vínculo ${ }^{4}$, cujas fundamentações são reforçadas no estudo de Sousa ${ }^{5}$ e reveladas as especificidades do modo de (re)organização da saúde brasileira, o que torna o conceito de atenção básica tanto completo quanto complexo, devido às diversas nuances que 0 compõem.

É a partir do fim da década de 70 e início da década de 80 que a atenção básica é ampla e efetivamente implantada no país, como uma medicina pobre voltada para as camadas sociais mais bai xas. Ganha-se em expansão de equipamentos de saúde em uma época pródiga em gastos na área, principalmente pela assistência centrada nos hospitai $\mathrm{s}^{3,6}$. M as na primeira metade da década de 90 , surgem programas governamentais que buscam modificar a forma de fazer atenção básica no país. São criados o Programa de Agentes Comunitários de Saúde (PACS) e o Programa de Saúde da Família (PSF); este último se consolida como estratégia reorganizadora do sistema de saúde nacional, recém-aprovado na constituição de 1988 e editado nas Leis Orgânicas da Saúde (Leis n 8.080 en $\left.^{\circ} 8.142\right)^{7,8}$.

Mais recentemente, observamos uma preocupação das esferas de governo para que haja uma reorientação do modelo de atenção à saúde no País, por meio do apoio à Atenção Básica e do estímulo à adoção da estratégia de Saúde da Família como estruturante para a organização dos sistemas municipais de saúde, e também uma ampliação e consolidação da estratégia Saúde da Família, com orientação para organização dos serviços que considere a incorporação de novos cenários epidemiológicost.

Entretanto, mesmo com a orientação de busca denovos cenários epidemiológicos, observa-se uma forte centralização na forma de se produzir atenção básica no país, através da verticalização dos programas eações a partir do nível central de gestão ${ }^{6}$. Considerando ainda que os dados e indicadores em atenção básica têm suas limitações ${ }^{9} \mathrm{e}$ que uma ação integral necessita de dados de saúde das populações para ser planejada ${ }^{10,11}$, há de se observar uma lacuna deixada entre a demanda real da população e a demanda programada através dos programas verticais, como por exemplo o programa para hipertensos e diabéticos ${ }^{11,12}$.

Os limites dos dados e indicadores na atenção básica podem ser evidenciados pela subnotificação dedisfunções musculoesqueléticas, sobretudo quando a Organização M undial da Saúde (OM S) ${ }^{13}$ promove a "Década do Osso e da Articulação (2000-2010)", sendo este movimento responsável pela iniciativa de alguns países em pôr em pauta a saúde musculoesquelética da população ${ }^{14}$.

M esmo diante dessas iniciativas, nenhum instrumento de coleta de dados nos programas PACS e PSF insere a saúde osteoarticular como um possível cenário de intervenção programática, seja ela de promoção à saúde, seja de prevenção de doenças ou reabilitação ${ }^{9}$. Dessa forma, questiona-se o quão integral é a atenção básica oferecida à população brasileira, já que este poderia ser um potencial cenário de novas intervenções de saúde primária.

\section{A saúde musculoesquelética ea atenção básica à saúde}

Entreos problemas mais comuns enfrentados pela população brasileira e mundial e que aqui são subnotificados na atenção básica, podemos destacar os distúrbios osteomusculares relacionados ao trabalho (D ort), as dores lombares e diversas outras disfunções musculoesqueléticas, sendo essas as principais expressões de doenças como artrite e a osteoartrose. As primeiras podem gerar diferentes graus deincapacidadefuncional, sendo consideradas um dos mais graves problemas no campo da saúde do trabalhador ${ }^{15}$. Reis et al. ${ }^{16}$ mostraram em seu estudo que as doenças musculoesqueléticas passaram a ser a principal causa de adoecimento no trabalho, sendo que dos dez diagnósticos mais prevalentes, nove eram doenças musculoesqueléticas. Esses distúrbios são responsáveis pela maior parte dos afastamentos do trabal ho e pelos custos com pagamentos de indenizações, tanto no Brasil como na maior parte dos países industrializados ${ }^{16}$.

As doenças reumáticas, por sua vez, podem ser agudas, recorrentes ou crônicas, atingindo 
pessoas detodas as idades. Como causa frequente de incapacidade, as doenças reumáticas são, no seu conjunto, as principais responsáveis pelos custos com a saúde, quer sejam el es diretos, como consultas, medicamentos ou cuidados de reabilitação, quer sejam indiretos ${ }^{14}$.

Já em relação às dores lombares, em alguma época da vida, de $70 \%$ a $85 \%$ detodas as pessoas sofrerão desse mal ${ }^{17}$. Além disso, a lombalgia é causadora de licenças médicas, elevados custos com saúde, comprometimento da produtividade por alto absentismo ocupacional ${ }^{18}$. A prevalência de dor lombar crônica éimportante quando se considera a quantidade de limitação das atividades e de demanda por serviço de saúde que esse problema gera' ${ }^{19}$.

Percebe-se que os distúrbios musculoesque léticos são predominantes e seus impactos são profundos nos indivíduos, nos sistemas de saúde e nos sistemas de assistência social. Eles são a maior causa de dor severa e persistente e deincapacidade física, afetando centenas de milhões de pessoas em todo o mundo. Segundo Woof ePfleger $^{20}$, essas dores afetam significativamente 0 estado psicossocial dosindivíduos acometidos, tanto quanto das suas famílias e carreiras profissionais. Os agravos musculoesqueléticos constituem-se num grupo diverso, apresentando fisiopatologia variada, ligados intrinsecamente pela associação entre dor ediminuição da função físi$\mathrm{ca}^{20}$. Além disso, a dor e a incapacidade física trazem com as disfunções musculoesqueléticas, efeitos na função social e saúde mental, além de depreciar a qualidade de vida do paciente ${ }^{20}$.

M esmo sendo expressão direta deafastamento deuma condição saudável, a dor ea incapacidade funcional são elementos subnotificados pela atenção básica em saúde no Brasil, uma vez que não são registradas especificamente nas unidades básicas de saúde (UBS), sendo que uma das responsabilidades da estratégia de aten ção básica no Brasil é manter uma postura pró-ativa ante os problemas de saúde doença da população, além de desenvolver suas atividades de acordo com o planejamento e a programação realizados com base no diagnóstico situacional ${ }^{4}$. Estudos sobre incapacidade funcional para o trabalho, dor lombar crônica e outras disfunções musculoesqueléticas crônicas têm mostrado a alta incidência desses problemas na população mundial ${ }^{15-20}$.

É necessário ressaltar que, apesar de os diversos estudos supracitados indicarem grandeincidência de distúrbios que hoje não possuem dado pró-ativo na atenção básica, poucos são os que fazem o levantamento in loco, dentro das comunidades, pois a família, o círculo social eas redes de relaçõe ${ }^{21}$ e convivência que ali se inserem desempenham um papel importante em todo o processo que vai do sentir-se saudável, sentir necessidade de buscar o serviço de saúde, obter os cuidados necessários, sentir-se bem acompanhado pelo serviço desaúdee, novamente, sentir-se saudável${ }^{22}$.

Essa escassez de estudos na atenção básica dificulta a identificação de necessidades desaúde da população e o subsequente planejamento de atividades que venham a supri-las. M esmo com o crescimento da cobertura da atenção básica no Brasil, na prática ainda encontramos um modelo hegemônico bastante ligado ao tecnicismo no modo de operar o Sistema Ú nico de Saúde. Esse modelo promove, dentre outras coisas, a submissão dos processos decisórios sobre a organização do sistema de saúde à busca da padronização do processo saúde-doença, à focalização das intervenções e ao arranjo dicotômico entre "cestas" de ações básicas e filas para serviços altamente especializados ${ }^{22}$.

Levando isso em consideração, verificamos que apenas com assistência não seria possível suprir o cuidado ea atenção que exigem as diferentes morbidades. Por isso, a integralidade deve estar presente nas práticas de atenção, nos princípios da gestão ena organização das políticas de saúde. Esses argumentos mostram que a "cesta" de procedimentos baseados na "lista" de indicadores fechados não é suficiente para produzir uma atenção básica à saúde que seja abrangente, integral e resolutiva para a maioria dos problemas da população ${ }^{23}$.

Nessa perspectiva, este estudo se propõe a fornecer dados primários sobre a situação de saúde osteoarticular de uma população adscrita a uma UBS, entremeando o conceito de integralidade na saúde com algumas variáveis poucas vezes pesquisadas pelas equipes de atenção básica. Neste sentido, o estudo se propõe ainda a questionar a real aplicação de ações integrais no seio da atenção básica brasileira.

\section{M etodologia}

Este estudo se caracteriza por ser descritivo, registrando, analisando e correlacionando dados sobre a saúde osteoarticular de uma população sem, no entanto, manipular as variáveis ${ }^{24}$. Consistiu de duas etapas previamente definidas. $\mathrm{Na}$ primeira etapa, foram aplicados questionários com pessoas que procuram o serviço da unidade 
básica de saúde do bairro das Rocas, em $\mathrm{N}$ atal (RN) (demanda espontânea). A segunda etapa consistiu na aplicação dos questionários nos domicílios (dados pró-ativos). A escolha desses dois lócus da pesquisa buscou mimetizar o trabalho de um profissional de atenção básica.

A pesquisa foi realizada no período de outubro de2006 a junho de 2007 no bairro das Rocas, pertencente ao distrito sanitário Leste da cidade de Natal. Este bairro foi escolhido por apresentar uma estrutura de saúde com equipes de PSF, o que contribuiu para a delimitação do âmbito da pesquisa e para o acesso aos residentes dos domicílios.

$\mathrm{Na}$ primeira etapa, todas as pessoas que che garam à unidade foram abordadas e questionadas sobre o motivo da consulta, formando um grande grupo (N1). A amostra da UBS foi constituída de todos os entrevistados (n1) que foram identificados no grande grupo (N1) com queixas de dor osteomioarticular. 0 número de indivíduos do grande grupo (N1) foi de duzentas pessoas. 0 número de entrevistados ( $\mathrm{n} 1$ ) foi variável, de acordo com a sua queixa; neste caso, os entrevistados apresentavam algum tipo de dor osteomioarticular. Ainda na primeira etapa, cada indivíduo do grupo (n1) foi novamente contatado após a consulta para se identificar qual o encaminhamento médico dado para aquele caso.

A população da segunda etapa foi delimitada por amostragem, a partir das áreas da Estratégia de Saúde da Família do bairro; das quatro áreas que compõem a cobertura da UBS, duas foram sorteadas para a pesquisa em domicílios.

A amostra foi composta por todos os indivíduos que residiam dentro da área selecionada, que possuíam sintomasosteomioarticulareseque estavam classificados nos critérios deinclusão. Os domicílios foram selecionados de forma alternada (a família residente em uma casa era entrevistada e aseguinte não participava da entrevista), a partir das quadras onde os agentes comunitários desaúde fossem realizar visitas nos dias de coleta.

As visitas domiciliares caracterizaram-se como busca pró-ativa dos dados epidemiológicos nas duas áreas do PSF previamente selecionadas, respeitando os critérios de seleção dos sujeitos anteriormentedescritos. Osindivíduos eram questionados quanto à referência de dor nos últimos sete dias; caso respondessem que "sim", eram questionados sobrea característica da dor referida, sendo que somente as dores osteoarticulares permitiam o preenchimento do questionário completo.

Como critérios de inclusão foram considerados indivíduos com idade igual ou superior a 20 anos na data da entrevista, que não foram víti- mas de acidente vascular encefálico e/ou demais patologias que acarretassem déficits funcionais, excluindo doenças osteomusculares. Das pessoas que chegavam à UBS, não foram considerados demanda espontânea - por conseguinte, foram excluídos da pesquisa - os indivíduos que participam do programa de diabéticos e hipertensos, mulheres que iriam realizar ou apresentar exames preventivos e gestantes.

0 instrumento de coleta utilizado para o levantamento epidemiológico foi um roteiro que inseriu a Escala Visual Analógica (EVA) de dor, questões sociodemográficas, uma sequência de questões para caracterização da dor, questões sobre a relação trabalho/dor osteoarticular e a partedeFunção Física eFunção dos Papéis do SF-36.

É relevante ressaltar que, para participar da pesquisa, todos os sujeitos assinaram o Termo de Consentimento Livre e Esclarecido e que este estudo foi previamente aprovado pelo Comitê de Ética em Pesquisa do Hospital Universitário Onofre Lopes (CEP-HUOL).

Os dados coletados foram organizados, tabulados e submetidos a uma análise estatística por meio da estatística descritiva visando quantificar e, por conseguinte, caracterizar as amostras investigadas. Concomitantemente, os achados foram confrontados com a literatura, no intuito de aprofundar a temática.

\section{Resultadosediscussão}

\section{Dados sociodemográficos e ocupação e funcionalidade}

Dos dados referentes à população da unidade básica de saúde, encontramos uma distribuição por sexo com maioria de mulheres nos grupos. No grupo que foi abordado inicialmente ( $N 1=200$ ), o número de mulheres foi equivalente a $79 \%$ da amostra. Já no grupo que referiu queixa de dor osteoarticular (n1=64), a porcentagem foi de $84,37 \%$ do sexo feminino.

Dados semelhantes foram encontrados na pesquisa em domicílios, em que do total de entrevistados ( $\mathrm{N} 2=186$ ) o número de mulheres foi equivalentea $68,82 \%$, esomente dos sujeitos que referiram dor nos últimos sete dias (n2=48), $81,25 \%$ eram do sexo feminino. Em relação à ocupação atual dos que responderam ao questionário na U BS, $42,18 \%$ eram donas de casa. N os domicílios, essa porcentagem foi de $52,08 \%$.

A média de idade foi de 59,78 anos $( \pm 15,33)$ no grupo da UBS (n1=64) e de 56,91 anos ( \pm 14,34 ) nos entrevistados em domicílio (n2=48). 
Setomarmos os resultados do perfil da amostra, teremos uma predominância do sexo feminino na queixa de dor osteoarticular, o quejá foi demonstrado com diversos estudos de disfunções musculoesqueléticas, no trabalho ou por doenças reumáticas ${ }^{15,16,25,26}$. Também se observa uma média de idade próxima à faixa dos 60 anos nos grupos da U BS e dos domicílios, enos domicílios a média geral foi de 46,54 anos, sugerindo que as pessoas que se queixaram de dor têm média de idade superior. Idades mais avançadas também estão relacionadas com problemas musculoesqueléticos ${ }^{26,27}$.

Em relação a quais atividades do dia a dia pioravam a dor osteoarticular dos entrevistados, na UBS $73,44 \%$ relataram piora da dor quando faziam atividades relacionadas ao trabalho. Nos domicílios, esse número foi de $62,5 \%$ (Tabela 1 ).

A grande maioria dos entrevistados, em ambos os grupos, tem sua queixa de dor aumentada com atividades relacionadas ao trabalho, ou seja: as donas de casa, que são ampla maioria no estudo, sentem piora nas dores quando realizam atividades como lavar roupa, varrer casa, lavar louça ou fazer a faxina rotineira da casa. 0 estudo de Rosa et al. ${ }^{28}$ aponta que a ocupação de dona de casa está relacionada à diminuição da capacidade funcional. Torna-se evidente, então, uma atenção maior dos profissionais da atenção básica em relação à saúde dos trabalhadores que residem/trabalham nas áreas de cobertura do PSF, configurando um importante papel da atenção básica à saúde ${ }^{29}$. Arcanjo et al..$^{30}$ sugerem também uma atuação multiprofissional para enfrentamento de dores na coluna vertebral dosindivíduos, estudo que envolveu mulheres donas de casa que possuíam dores nas costas.

Dados adicionais foram pesquisados em re lação à capacidade funcional através das seções Função Física e Função dos Papéis do SF-36. Do total de entrevistados, somando-se U BS e domicílios ( $N=112$ ), mais da metade disseram que se sentiram limitados no trabalho no último mês por causa da sua saúde $(51,78 \%)$. Ainda do total deentrevistados, 66,07\% disseram se sentir muito limitados para realizar atividades vigorosas e 53,57\% disseram sentir essa grande limitação ao inclinar-se, abaixar-se ou ajoel har-se.

Ainda segundo Rosa et al. ${ }^{28}$, as ações de saúde genéricas são menos produtivas do que as ações preventivas para se reduzir a incapacidadefuncional, pois veremos quea prevenção se configura como um importante ponto para desenvolver a saúde das populações ${ }^{1}$. Neste estudo, os dados encontrados na função física e dos papéis suge- rem uma diminuição da capacidade para o trabalho e para a realização de atividades de vida diária, como levantar objetos pesados e inclinarse, abaixar-se ou ajoel har-se, nos indivíduos com queixas osteoarticulares. Por isso, ações de prevenção para essa população podem se constituir como benéficas para a saúde desses indivíduos.

\section{Dados de prevalência, frequência \\ e intensidade da dor}

A prevalência de dor osteoarticular na UBS foi de $32 \%$ das pessoas que procuraram atendimento, excluindo as pessoas que fazem parte de programas verticais específicos. O u seja, uma em cada três pessoas procuraram 0 atendimento médico da unidade das Rocas com queixa de dores osteoarticulares. Já na pesquisa nos domicílios, a prevalência de pessoas com esse tipo de dor foi de $25,8 \%$, ou seja, aproximadamenteuma de quatro pessoas abordadas em domicílio referiram queixa de dor osteoarticular nos últimos sete dias.

As prevalências de queixa osteoarticular deste estudo se assemelham a outro realizado em um país industrializado, em que os dados revelaram que $30 \%$ da população refere sintomas musculoesquel éticos ${ }^{14}$. No Brasil, um estudo com idosos mostrou uma prevalência de dor crônica ainda maior na população estudada, atingindo $51,44 \%$ da população ${ }^{27}$. É importante ressaltar que artigos pesquisados mostram a importância da atenção básica na atuação na diminuição da dor e das disfunções decorrentes de problemas osteoarticulares ${ }^{28-31}$.

A Escala Visual Analógica (EVA) de dor foi utilizada para a avaliação do nível da dor nos sujeitos entrevistados, para então se comparar com outros estudos e obter um parâmetro da intensidade de dor na população estudada. N essa escala crescente de zero a dez, o sujeito declara

Tabela 1. A tividades diárias que pioram a dor dos indivíduos na unidade básica de saúde e nos domicílios.

\begin{tabular}{|c|c|c|c|c|}
\hline & \multicolumn{2}{|c|}{ UBS } & \multicolumn{2}{|c|}{ Domicílio } \\
\hline & $\mathrm{n} 1$ & $\%$ & $\mathrm{n} 2$ & $\%$ \\
\hline Relacionadas ao trabalho & 47 & 73,44 & 30 & 62,5 \\
\hline Não relacionadas & 4 & 6,25 & 6 & 12,5 \\
\hline Ambas & 3 & 4,69 & 5 & 10,42 \\
\hline Nenhuma & 10 & 15,62 & 7 & 14,58 \\
\hline Total & 64 & 100 & 48 & 100 \\
\hline
\end{tabular}


qual o seu nível de dor. Na UBS, a análise dos dados mostrou que a referência de dor através da EVA apresentava uma mediana de 8 ( Q 25 =5 e $Q 75=9$ ). N os domicílios, a mediana coletada com a EVA foi de 6 (Q 25=4 e Q 75=8). Na soma das populações ( $N=112)$, temos uma mediana de 7 para a EVA (Q25=5 e Q75=9).

Outro dado pesquisado foi a frequência da dor nos indivíduos; foi encontrado que $75 \%$ das pessoas entrevistadas na UBS possuíam uma queixa de dor diária, enquanto esse número nos domicílios foi de 58,34\% (Tabela 2).

0 alto índice no escore da EVA - que sugere dores mais fortes -, se comparado a outros estudos que trabalharam com essa escala em disfunções musculoesqueléticas $25,29,32,33$, e o grande número de indivíduos com essa queixa diária podem orientar serviços de atenção básica para essa parcela da população, uma vez que há uma forte associação entre a dor e a baixa capacidade para o trabalho ${ }^{15}$.

Atividade física, estratégias de superação da dor e resolutividade dos serviços de atenção básica

No questionário aplicado, os sujeitos foram inquiridos acerca da prática de atividade física e do tipo de atividade praticada. Os dados da Ta-

Tabela 2. Frequência da queixa de dor das pessoas avaliadas na unidade básica de saúde e nos domicílios.

\begin{tabular}{|c|c|c|c|c|}
\hline & \multicolumn{2}{|c|}{ UBS } & \multicolumn{2}{|c|}{ Domicílio } \\
\hline & $\mathrm{n} 1$ & $\%$ & $\mathrm{n} 2$ & $\%$ \\
\hline Diária & 48 & 75 & 28 & 58,34 \\
\hline De 1 a 5 vezes na semana & 7 & 10,94 & 16 & 33,33 \\
\hline M enos de 1 a 3 vezes no mês & 7 & 10,94 & 3 & 6,25 \\
\hline Não sabe definir & 2 & 3,12 & 1 & 2,08 \\
\hline Total & 64 & 100 & 48 & 100 \\
\hline
\end{tabular}

Tabel a 3. Atividade física avaliada na unidade básica de saúde e nos domicílios.

\begin{tabular}{lrrrrr}
\hline & \multicolumn{2}{c}{ UBS } & & \multicolumn{2}{c}{ Domicílio } \\
\cline { 2 - 3 } \cline { 5 - 6 } & $\mathrm{n} 1$ & $\%$ & & $\mathrm{n} 2$ & $\%$ \\
\hline Sedentário & 40 & 62,65 & & 32 & 66,67 \\
Caminhada & 17 & 26,57 & & 10 & 20,83 \\
Outra atividade & 7 & 10,93 & & 6 & 12,5 \\
Total & 64 & 100 & & 48 & 100 \\
\hline
\end{tabular}

bela 3 mostram que $62,65 \%$ dos indivíduos entrevistadosnaU BS e66,67\% nosdomicílios eram sedentários.

0 grande número de indivíduos sedentários dentro da amostra da pesquisa vem corroborar a ideia de promoção à saúde e prevenção de doenças articulares eósseas, uma vez quea literatura revela queum dos principaisfatores queinfluenciam a saúde osteoarticular e a qualidade de vida éa prática de ati vidades físicas regulares ${ }^{34,35}$.

No entanto, ao analisar a Tabela 4 encontramosque, do total deentrevistados naU BS, $62,5 \%$ aliviam sua dor com medicamentos, enos domicílios, $79,17 \%$ aliviam a dor com esse recurso. Quando questionados sobre o que aliviava a dor, $17,19 \%$ das pessoas pesquisadas na U BS usavam exclusivamente medicamento para alívio da dor; já nos domicílios, esse número era de $12,5 \%$.

Os indivíduos que participaram da pesquisa utilizam medicamentos para o alívio da dor, contemplando a maioria dos entrevistados. Esse dado se torna relevante na medida em que se discutem os efeitos da medicalização na sociedade. M ais uma vez, destaca-se o papel da atenção básica na prevenção de doenças, promoção da saúde e no estímulo a alternativas populares e não medicalizantes para a obtenção de um meIhor estado de saúde ${ }^{1,36,37}$.

Um dado foi colhido somente entre os usuários da UBS que tinham queixa osteoarticular. Depois da consulta médica, procurou-se obter qual o encaminhamento dado pelo profissional, através de questionamento direto ao paciente. $\mathrm{A}$ Tabela 5 mostra que $25 \%$ dos usuários tiveram como encaminhamento a prescrição de medicamentos (14,06\% anti-inflamatórios e 10,94\% analgésicos), e 40,62\% das pessoas foram encaminhadas a especialistas.

Tabela 4. 0 que alivia a sua dor? Dados coletados na unidade básica de saúde e nos domicílios.

\begin{tabular}{lrrrrrr}
\hline & \multicolumn{2}{c}{ UBS } & & \multicolumn{2}{c}{ Domicílio } \\
\cline { 2 - 3 } \cline { 6 - 7 } & $n 1$ & $\%$ & & n2 & $\%$ \\
\hline Apenas medicamento & 11 & 17,19 & & 6 & 12,5 \\
M edicamento + outros & 29 & 45,31 & & 32 & 66,67 \\
recursos & & & & & \\
Não utiliza medicamento & 20 & 31,25 & & 7 & 14,58 \\
Nada/N ão sabe definir & 4 & 6,25 & & 3 & 6,25 \\
Total & 64 & 100 & & 48 & 100 \\
\hline
\end{tabular}


Tabela 5. Encaminhamentos dados pelos médicos na unidade básica de saúde.

\begin{tabular}{lrc}
\hline & \multicolumn{2}{c}{ UBS } \\
\cline { 2 - 3 } & $\mathrm{n} 1$ & $\%$ \\
\hline Anti-inflamatório & 9 & 14,06 \\
Ortopedista/Reumatologista & 18 & 28,12 \\
Radiografia/Densitometria & 15 & 23,44 \\
Analgésico & 7 & 10,94 \\
Especialista não relacionado à & 8 & 12,50 \\
queixa osteoarticular & & \\
\hline
\end{tabular}

Foi encontrado, portanto, um número de encaminhamentos a especialistas que vai ao encontro do que se espera da atuação em atenção básica à saúde, uma vez que a literatura considera queo nível deresolutividade da atenção básica esteja perto dos $80 \%$ dos casos, sendo admitido encaminhamento deapenas $20 \%$ da deman da 38,39 . M esmo com muitos casos diagnosticados que necessitam de acompanhamento específico, os dados deste estudo sugerem que há uma elevação do adoecimento na população estudada.

\section{A atenção básica diante dos resultados}

A literatura mundial mostra as vantagens dos serviços de saú de baseados em atenção primária à saúde em relação aos que se baseiam no cuidado através das especializações 2,10,39,40. Ações de promoção, preven ção e reabilitação devem estar próximas à população que as utiliza, sendo essas ações voltadas para as necessidades desses indivíduos, das famílias residentes na comunidade assistida ${ }^{1,2,4}$.

Schrai ber eM endes-Gonçalves ${ }^{11}$ definem três tipos de necessidades que podem existir em um serviço de atenção básica: (1) as que são historicamente constituídas e, por isso, são as que a população procura satisfazer através dos serviços de saúde; (2) as que não são sentidas pela população, mas identificadas pelos técnicos; (3) as necessidades que são descobertas no trabalho cotidiano dentro da comunidade, nas relações cotidianas, através da criação deespaços nos quais surjam necessidades que não são conhecidas pelos técnicos.

Estudando a organização da atenção básica no Brasil, observamos uma fortetendência à central ização/verticalização das ações, efetivadas através de programas ${ }^{6,23,41}$. Essas ações comprometem diretamentea emergência deespaços deescu- ta, em que as necessidades sejam identificadas a partir da participação dos próprios indivíduos.

0 que em um primeiro momento foi considerado benéfico para uma homogeneização da atenção em um país tão pleno de desigualdades regionais ${ }^{41}$ passa a ser questionado à medida que - SUS avança e se consolida e à medida que as diferenças entre centros urbanos e zona rural emergem com diferentes necessidades de saúde ${ }^{41}$.

$\mathrm{H}$ oje, a atenção básica é pautada pela centralização das ações, através de "cestas" de procedimentos, premiadas com vinculação do repasse de verbas com os dados prospectados nas unidades básicas ${ }^{23}, 0$ que pode trazer consequências negativas, uma vez que os profissionais aqui inseridos se restringem apenas aos dados solicitados centralmente pelo M inistério da Saúde, indo de encontro ao proposto pela ideia dos novos cenários epidemiológicos. São os novos cenários que levam à integralidade da atenção, com as necessidades reais da população sendo identificadas e, então, transformadas em ações que levem a um estado saudável as populações. Os dados deste trabalho mostram outro lado da saúde de uma população urbana que não está sendo explorado atualmente pela atenção básica, limitando as ações integrais de saúde sobre essa população.

Bodstein ${ }^{42}$ contrapõe a ideia de cestas de procedimentos dizendo queela não leva em consideração a proposta formulada pela Norma Operacional Básica-96 (NOB-96) como um todo e não valoriza a Programação Pactuada e Integrada (PPI) como um instrumento para a garantia do direito à assi stência à saúde integral. Se considerarmos a PPI como instância de pactuação de ações entre gestores, teremos novo elemento de crítica em relação à aplicação de ações integradas, uma vez quea saúde osteoarticular, por exemplo, não chega a ser pesquisada nas UBS, portanto, não devem ser levadas para pactuaçãa de ações em atenção básica se a gestão não possui dados para avaliação situacional ${ }^{43}$.

Além da responsabilidade da gestão na não assunção da integralidade nas ações da atenção básica, podemos destacar a responsabilidade dos profissionais nesse aspecto. Botti ${ }^{44}$ cita a internalização do pensamento normativo pela equipe, um modelo de assistência médico-centrado e a acessibilidade do sistema reduzida unicamente ao agendamento de consultas médicas como elementos que afastam os profissionais das necessidades reais de saúde da população e de uma boa prática de aten ção básica no PSF. Uma atuação profissional diferente nas UBS pode levar a uma 
escuta maior das necessidades dos indivíduos, fazendo com que as ações de saúde se aproximem cada vez mais dos problemas das populações eque novos cenários epidemiológicos sejam descobertos.

A efetivação de uma atenção básica à saúde passa pelo compromisso de todos para a assunção da responsabilidade de promover cada um dos pontos que compõem a atenção básica. N esse sentido, cabe ressaltar a importância da equipemultiprofissional como provedora deintegralidade ${ }^{45}$. Outro ponto muito discutido, porém pouco efetivado no Brasil, é a montagem dessa equipe multiprofissional, em que pese o comprometimento deações verdadeiramenteintegrais no seio da atenção básica à saúde ${ }^{6}$.

Por fim, há de se destacar que a identificação da integral idade às respostas governamentais a de terminados problemas de saúde ou às necessi dades de grupos específicos levaria à definição de políticas de atenção enão mais s programas de assistên$\mathrm{Cia}^{22}$. É isso que os dados deste trabalho suge rem: que a identificação de necessidades locais, no caso problemas osteoarticulares, possa contribuir para uma política de atenção em que se considere a integração dos níveis de assistência, sobretudo o que se propõe a ser mais resolutivo, com menor custo ecom maior afinidade com as famílias e indivíduos, que é a atenção básica.

\section{Conclusão}

Os dados deste estudo se constituem como ele mentos iniciais para revelar a saúde osteoarticular de uma população adscrita a uma unidade básica de saúde (UBS). Eles sugerem que dor e funcionalidade são elementos pouco analisados na atenção básica à saúde, apesar de serem reflexo direto de saúde e de influenciarem as atividades de vida diária e laborais dos indivíduos, afe tando assim a qualidade de vida.

Assim, espera-se que os serviços de saúde voltem suas ações para esses problemas: primeiro, identificando, no cotidiano do trabalho, ouvindo indivíduos efamílias cobertas pela atenção básica brasileira, esses problemas; depois, tentando resolvêlos de forma criativa no trabalho vivo, enfocando, sobretudo, as ações de promoção e prevenção para evitar um adoecimento maior desses indivíduos.
Na Década do Osso e da Articulação (20002010), os gestores de saúde devem observar a prevalência da dor osteoarticular, que neste estudo chegou a ser de um em cada três indivíduos pesquisados, identificar os grupos de risco como as mulheres donas de casa com maior idade e planejar ações efetivas, em especial na porta de entrada do sistema de saúde brasileiro, que é a atenção básica.

Para isso, também se faz necessária uma mudança nos paradigmas da atuação profissional no Programa de Saúde da Família, a mudança do modelo médico-centrado para a incorporação de novos profissionais na equipe multiprofissional que deem resposta aos problemas musculoesqueléticos da população, como o educador físico e o fisioterapeuta - bem como a mudança na forma de trabalho, pois as equipes de PSF não devem reduzir os seus compromissos com a população a agendamentos de consulta, mas sim aproximar-se realmente da população coberta, ouvindo seus anseios e necessidades, pois muitos deles não estão sendo identificados pelo sistema de saúde para planejamento, monitoramento e avaliação das ações, como é 0 caso da saúde osteoarticular.

\section{Colaboradores}

MS M ata planejou e elaborou a pesquisa, organizou e redigiu o texto; FA Costa acompanhou a pesquisa, orientou a organização do texto e participou da revisão; TO Souza colaborou na elaboração da metodologia; ANS M ata e JF Pontes contribuíram na revisão final do texto. 


\section{Referências}

1. Declaração de Alma-Ata. Conferência Internacional sobre Cuidados Primários de Saúde. Alma Ata; 1978. [acessado 2006 dez 20]. Disponível em: http:// www.opas.org.br/coletiva/uploadArq/Alma-Ata.pdf

2. Atun R. What are the advantages and disadvantages on restructuring a health care system to be more focused on primary care services? Copenhagen: WHO Regional Office for Europe; 2004. [acessado 2007 mar 20]. Disponível em: http://www.euro.who.int/ document/e82997.pdf

3. Sampaio LFR. 0 papel das secretarias estaduais de saúde no acompanhamento e avaliação da atenção primária à saúde: contribuições para discussão. In: Conselho Nacional de Secretários de Saúde. Acompanhamento e avaliação da atenção primária. Brasília: Conass; 2004. p. 21-41. (Conass Documenta, n 7).

4. Brasil. Política Nacional de Atenção Básica. Brasília: M inistério da Saúde; 2006. (Série Pactos pela Saúde, v. 4).

5. Sousa MF. Programa Saúde da Família no Brasil: uma agenda incompleta? Cien Saude Colet 2009; 14(Supl. 1):1325-1335.

6. Aciole GG. A saúde no Brasil: cartografias do público e do privado. São Paulo: Hucitec; 2006.

7. Brasil. Saúde da Família no Brasil: uma análise de indicadores selecionados: 1998-2004. Brasília: Ministério da Saúde; 2006. p. 12.

8. Marques RM, M endes A. Atenção básica e o Programa Saúde da Família (PSF): novos rumos para a política de saúde e seu financiamento? Cien Saude Colet 2003; 8(2):403-415.

9. Brasil. SIAB: manual do sistema de informação de atenção básica. Brasília: M inistério da Saúde; 1998.

10. Macinko J, M ontenegro $\mathrm{H}, \mathrm{N}$ ebot Adell $\mathrm{C}$, Etienne C y Grupo de Trabajo de Atención Primaria de Salud de la Organización Panamericana de la Salud. La renovación de la atención primaria de salud em las Américas. Rev Panam Salud Publica 2007; 21(2/3):73-84.

11. Schraiber LB, M endes-Gonçalves RB. Necessidades de saúde e atenção primária. In: Schraiber LB, Nemes MIB, M endes-Gonçalves RB, organizadores. Saúde do adulto: programas e ações na unidade básica. São Paulo: Hucitec; 2000. p. 29-47.

12. Teixeira CF. Promoção e vigilância da saúde no contexto da regionalização da assistência à saúde no SUS. Cad Saude Publica 2002; 18(Supl.):153-162.

13. Garfin SR, Andersson G, Gronblad M, Rydevik B. The Bone and Joint Decade, 2000-2010, for Prevention and Treatment of Musculoskeletal Disorders. Spine 1999; 24(11):1055.

14. Portugal. Programa Nacional contra as Doenças Reumáticas. [acessado 2007 jan 12]. Disponível em: http://www.myos.pt/downloads/circularpncdoencas reumaticas.pdf

15. Walsh IAP, Corral S, Franco RN, Canetti EEF, Alem MER, Coury HJCG. Capacidade para o trabalho em indivíduos com lesões músculo-esqueléticas crônicas. Rev Saude Publica 2004; 38(2):149-156.
16. Reis RJ, Pinheiro TMM, Navarro A, Martin M M . Perfil da demanda atendida em ambulatório de doenças profissionais e a presença de lesões por esforços repetitivos. Rev Saude Publica 2000; 34(3):292-298.

17. Andersson G. Epidemiological features of chronic low-back pain. Lancet 1999; 354:581-585.

18. Cid VV, Jiménez ED. Participación del kinesiólogo en un programa de prevención de problemas lumbares. Revista Kinesiologia 1991; 29:259-264.

19. Silva MC, Fassa AG, Valle NCJ. Dor lombar crônica em uma população adulta do Sul do Brasil: prevalência e fatores associados. Cad Saude Publica 2004; 20(2):377-385.

20. Woolf AD, Pfleger B. Burden of major musculoskeletal conditions. Bulletin of he World Health Organization 2003; 81:646-656.

21. Gerhardt TE. Itinerários terapêuticos em situações de pobreza: diversidade e pluralidade. Cad Saude Publica 2006; 22(11):2449-2463.

22. Brasil. Curso de Formação de Facilitadores de Educação Permanente em Saúde: unidade de aprendizagem. In: Brasil. Ministério da Saúde. Fundação Oswaldo Cruz. Análise do contexto da gestão e das práticas de saúde. Rio de Janeiro: Ministério da Saúde/Fiocruz; 2005. p. 88-100.

23. Bueno WS, M erhy EE. Os equívocos da NOB 96: uma proposta em sintonia com os projetos neoliberalizantes? [acessado 2007 abr 5]. Disponível em: http:/ /www.datasus.gov.br/cns/temas/N O B96/N O B96 crit.htm

24. Cervo AL, Bervian PA. M etodologia científica. São Paulo: Prentice Hall; 2002.

25. Chahade W, Samara AM, Silva NA, Seda H, Radominski SC, Keiserman M, Duarte A, Goldensteins L, Gonçalves H, Ximenes AC. Eficácia sintomática dos insaponificáveis de abacate e soja (IAS) no tratamento de osteoartrose (OA) de quadril e joelho. Rev Bras M ed 2004; 61(11):711-718.

26. Birrel F, Afzal C, Nahit E, Lunt M, MacFarlane JG, Cooper C, Croft PR, Hosie G, Silman AJ. Predictors of hip joint replacement in new attenders in primary care with hip pain. Br J Gen Pract 2003; 53(486):26-30.

27. Dellaroza M SG, Pimenta CAM, M atsuo T. Prevalência e caracterização da dor crônica em idosos não institucionalizados. Cad Saude Publica 2007; 23(5):1151-1160.

28. Rosa TEC, Benício MHD, Latorre MRDO, Ramos LR. Fatores determinantes da capacidade funcional entre idosos. Rev Saude Publica 2003; 37(1):40-48.

29. Nogueira DP. Incorporação da saúde ocupacional à rede primária de saúde. Rev Saude Publica 1984; 18:495-509.

30. Arcanjo GN, Silva RM, Nations MK. Saber popular sobre dores nas costas em mulheres nordestinas. Cien Saude Colet 2007; 12(2):389-397.

31. Watkin $\mathrm{H}$. Back pain: an integrated approach in primary care. Acupuncture in M edicine 2004; 22(4): 203-206. 
32. Furtado RNV, Carazzato S, Farias CA, Chamlian $T R, M$ asiero D. Síndrome miofascial: comparação entre 0 tratamento com infiltração de trigger points e medicação oral (ciclobenzaprina). Acta Fisiátrica 2002; 9(3):117-126.

33. Briganó JU, Macedo CSG. Análise da mobilidade lombar e influência da terapia manual e cinesioterapia na lombalgia. Semina: Ciências Biológicas e da Saúde 2005; 26(2):75-82.

34. Toscano JJO. Atividade física e qualidade de vida relacionada à saúde de idosos do sexo feminino [dissertação]. Aracaju: Núcleo de Pós-Graduação em Medicina, U niversidade Federal de Sergipe; 2005.

35. Matsudo SM, Matsudo VKR, Barros N eto TL. Atividade física e envelhecimento: aspectos epidemiológicos. Rev Bras M ed Esporte 2001; 7(1):2-13.

36. Peters JT, Sanders C, Dieppe P, Donovan J. Factors associated with change in pain and disability over time: a community-based prospective observational study of hip and knee osteoarthritis. Br J Gen Pract 2005; 55(512):205-211.

37. Buss PM. Promoção da saúde e qualidade de vida. Cien Saude Colet 2000; 5(1):163-177.

38. Takeda S. Organização de serviços de atenção primária à saúde. In. Duncan BB, Schmidt MI, Giugliani ERJ. M edicina ambulatorial: condutas de atenção primária baseadas em evidências. Porto Alegre: Artmed; 2004.

39. Starfield B. Is primary care essential? Lancet 1994; 344:1129-1133.

40. Shi L, Starfield B, Kennedy B, Ichiro K. Income inequality, primary care, and health indicators. Journal of Family Practice 1999; 48:275-284.

41. Levcovitz E, Lima LD, Machado CV. Política da Saúde nos anos 90: relações intergovernamentais e o papel das Normas Operacionais Básicas. Cien Saude Colet 2001; 6(2):269-291.

42. Bodstein R. Atenção básica na agenda da saúde. Cien Saude Colet 2002; 7(3):401-412

43. Felisberto E. Monitoramento e avaliação na atenção básica: novos horizontes. Rev Bras Saúde Matern Infant 2004; 4(3):317-321.

44. Botti ML, Scochi MJ. O aprender organizacional: relato de experiência em uma unidade básica de saúde. Saúde e Sociedade 2006; 15(1):107-114.

45. Mattos RA. Os sentidos da integralidade: algumas reflexões acerca de valores que merecem ser defendidos. In: Pinheiro R, M attos RA, organizadores. Os sentidos da integralidade na atenção e no cuidado à saúde. Rio de Janeiro: U erj/Abrasco; 2003.

Artigo apresentado em 31/10/2007

Aprovado em 12/11/2007

Versão final apresentada em 12/12/2007 